\title{
The Superior Parietal Lobule of Macaque Monkey: Relative Influence of Gaze and Static Arm Position during Reaching
}

\author{
DMarina De Vitis, ${ }^{1, *}$ Marta Tabanelli, ${ }^{1, *}$ CRossella Breveglieri, ${ }^{1}$ Matteo Filippini, ${ }^{1}$ Claudio Galletti, ${ }^{1}$ and \\ (1)Patrizia Fattori ${ }^{1,2}$
}

https://doi.org/10.1523/ENEURO.0362-21.2021

${ }^{1}$ Department of Biomedical and Neuromotor Sciences, University of Bologna, 40126 Bologna, Italy and ${ }^{2}$ Alma Mater Research Institute for Human-Centered Artificial Intelligence (Alma Human Al), University of Bologna, 40121 Bologna, Italy

\begin{abstract}
The superior parietal lobule (SPL) integrates somatosensory, motor, and visual signals to dynamically control arm movements. During reaching, visual and gaze signals are used to guide the hand to the desired target location, while proprioceptive signals allow to correct arm trajectory, and keep the limb in the final position at the end of the movement. Three SPL areas are particularly involved in this process: V6A, PEc, PE. Here, we evaluated the influence of eye and arm position on single neuron activity of these areas during the holding period at the end of arm reaching movements, when the arm is motionless and gaze and hand positions are aligned. Two male macaques (Macaca fascicularis) performed a foveal reaching task while single unit activity was recorded from areas V6A, PEc, and PE. We found that at the end of reaching movements the neurons of all these areas were modulated by both eye position and static position of the arm. V6A and PEc showed a prevalent combination of gaze and proprioceptive input, while PE seemed to encode these signals more independently. Our results demonstrate that all these SPL areas combine gaze and proprioceptive input to provide an accurate monitoring of arm movements.
\end{abstract}

Key words: gaze; posterior parietal cortex; proprioception; reaching movements; superior parietal lobule; visuomotor integration

\section{Significance Statement}

This study shows that the SPL areas V6A, PEc, and PE combine eye and static arm positions signals to build an estimate of the limb posture at the end of a reaching movement. The degree of integration of gaze and proprioceptive information changes from a joint processing of these signals in the caudal-most areas V6A and PEc (Brodmann area 7), to a more independent encoding in PE (Brodmann area 5). Our results support the existence of a functional trend in the SPL, with the anterior part dealing mainly with limb representation based on proprioception and the posterior one linking gaze and arm position signals for encoding reaching.

\section{Introduction}

Eye-hand coordination is a basic function that allows primates to interact with the surrounding environment. In goal directed arm movements, we first direct the gaze to the target, then move the limb toward it and, at the end of

Received September 7, 2021; accepted October 26, 2021; First published December 3, 2021.

The authors declare no competing financial interests. movement, hold the arm in the final desired position to allow target touching, pushing, grasping, or manipulation. The basis of these abilities is the integration of eye position with proprioceptive signals from the limbs, an

Author contributions: R.B., C.G., and P.F. designed research; M.D.V., R.B., and M.F. performed research; M.D.V. and M.F. contributed unpublished reagents/analytic tools; M.D.V. and M.T. analyzed data; M.D.V., M.T., R.B., M.F., C.G., and P.F. wrote the paper. 
operation that typically occurs in the superior parietal lobule (SPL; Battaglia-Mayer et al., 2001; McGuire and Sabes, 2011; Granek et al., 2012; Fattori et al., 2017). The SPL areas V6A, PEc, and PE (Fig. 1; Pandya and Seltzer, 1982; Galletti et al., 1999) are particularly involved in this process (Galletti and Fattori, 2003; Gamberini et al., 2011, 2018, 2020; Fattori et al., 2017). PE is an area rich in somatosensory cells which receives proprioceptive input from the limbs and is involved in the monitoring of limb posture and movement. It contains mainly neurons sensitive to joint rotations, activated by passive movements of the arm, but also cells spatially tuned by active reaching movements, suggesting that $\mathrm{PE}$ is mainly involved in the somatosensory monitoring of arm state during reaching (Duffy and Burchfiel, 1971; Sakata et al., 1973; Mountcastle et al., 1975; Georgopoulos et al., 1984; Kalaska et al., 1990; Kalaska, 1996; Gardner et al., 2007; De Vitis et al., 2019). PEc hosts somatosensory and visual neurons, as well as bimodal (somatovisual) cells modulated by arm movement and visual stimulation, and cells modulated by the direction of gaze. PEc is suggested to use these signals to perform a visuo-somatomotor control of reaching (BattagliaMayer et al., 2001; Ferraina et al., 2001; Breveglieri et al., 2006, 2008; Hadjidimitrakis et al., 2015; Piserchia et al., 2017; Gamberini et al., 2018). V6A is a visuomotor area that hosts neurons with sensory features comparable to those of PEc, but with a higher proportion of visual neurons (Gamberini et al., 2011, 2018) and of cells modulated by the direction of gaze (Galletti et al., 1995), but less somatosensory cells than PEc (Breveglieri et al., 2002). Therefore, in both PEc and V6A, somatosensory and gaze stimuli are used for reaching control but the degree of independent encoding of these signals in the two areas is still under debate.

In this article, we have investigated how arm-related and gaze-related signals influence single neurons of these areas by looking at the activity of these neurons at the end of a Fixation-to-Reach task, when both gaze and arm were stationary in space and time. The results showed that V6A, PEc, and PE neurons were differently modulated by gaze and arm-related signals. Area PE was more sensitive to limb proprioceptive input while PEc and V6A were more sensitive to proprioceptive and gaze signals, with

This work was supported by MAIA project, which has received funding from the European Union's Horizon 2020 research and innovation programme under grant agreement No 951910. This article reflects only the author's view and the Agency is not responsible for any use that may be made of the information it contains.

${ }^{*}$ M.D.V. and M.T. contributed equally to this work.

Acknowledgements: We thank Dr. Michela Gamberini and Dr. Lauretta Passarelli for the anatomical reconstructions and Massimo Verdosci and Francesco Campisi for technical assistance.

Correspondence should be addressed to Rossella Breveglieri at rossella. breveglieri@unibo.it.

https://doi.org/10.1523/ENEURO.0362-21.2021

Copyright $@ 2021$ De Vitis et al.

This is an open-access article distributed under the terms of the Creative Commons Attribution 4.0 International license, which permits unrestricted use, distribution and reproduction in any medium provided that the original work is properly attributed. the latter particularly modulated by the interaction between gaze and hand position.

\section{Materials and Methods}

\section{Experimental procedures}

Two male macaque monkeys (Macaca fascicularis), weighing 4 and $4.6 \mathrm{~kg}$, were involved in this study. The experiments were performed in accordance with the guidelines of EU Directives (86/609/EEC; 2010/63/EU) and Italian national laws (D.L.116-92, D.L. 26-2014) on the protection of animals used for scientific purposes. Protocols were approved by the Animal-Welfare Body and from the Italian Ministry of Health. During training and recording sessions, particular attention was paid to any behavioral and clinical signs of pain or distress.

\section{The Fixation-to-Reach task}

The animal sat in a primate chair (Crist Instruments) and performed a Fixation-to-Reach task. During this task, the monkey sat in front of a horizontal panel located at eye level with nine light-emitting diodes (LEDs; $6 \mathrm{~mm}$ in diameter) placed at different distances and directions used as fixation and reaching targets (Fig. 2). Since the targets were aligned at eye level, they could potentially obscure each other. We got the problem solved by gradually masking the LEDs, going from the thinner nearest targets to the ticker farthest one. Thus, the monkeys were able to easily discriminate them. The task was performed in darkness with the hand contralateral to the recording site. In the starting position, the monkey kept its hand on a button [hereafter called the home button (HB), $2.5 \mathrm{~cm}$ in diameter] placed $4 \mathrm{~cm}$ in front of the chest, outside the animal's field of view (Fig. 2). Target LEDs were arranged in three rows: one central, along the sagittal midline, and two laterals, at version angles of $-15^{\circ}$ and $+15^{\circ}$, respectively. Along each row, three LEDs were located at different depth, at vergence angles of $17.1^{\circ}, 11.4^{\circ}, 6.9^{\circ}$. The nearest targets were located at $10 \mathrm{~cm}$ from the eyes, whereas the LEDs placed at intermediate and far positions were located at 15 and $25 \mathrm{~cm}$, respectively (Fig. 2). Target positions were chosen to be all within the peripersonal space.

A trial began when the monkey pressed the HB (Fig. 2, HB press). After $1 \mathrm{~s}$, one of the nine LEDs was switched on to green and the monkey had to fixate the LED while keeping the HB button pressed (Fig. 2, LEDon). Then, the monkey had to wait 1.5-2.5 s for a change in the color of the same LED (from green to red) without performing any eye or arm movement (Fig. 2, epoch FIX). The color change was the go signal for the animal to release the HB and start an arm movement toward the target. Once reached the target, the animal was required to hold the hand on it for 0.8-1.2 s (Fig. 2, epoch HOLD). Target switching off cued the monkey to release it and return to the $\mathrm{HB}$, which ended the trial and allowed the monkey to receive its reward. Note that during FIX the monkey maintained the gaze still on one of the nine LEDs while the arm was located near the body because the hand was pressing the HB; during HOLD, the animal maintained fixation on the target LED while pushing it, so the arm was 


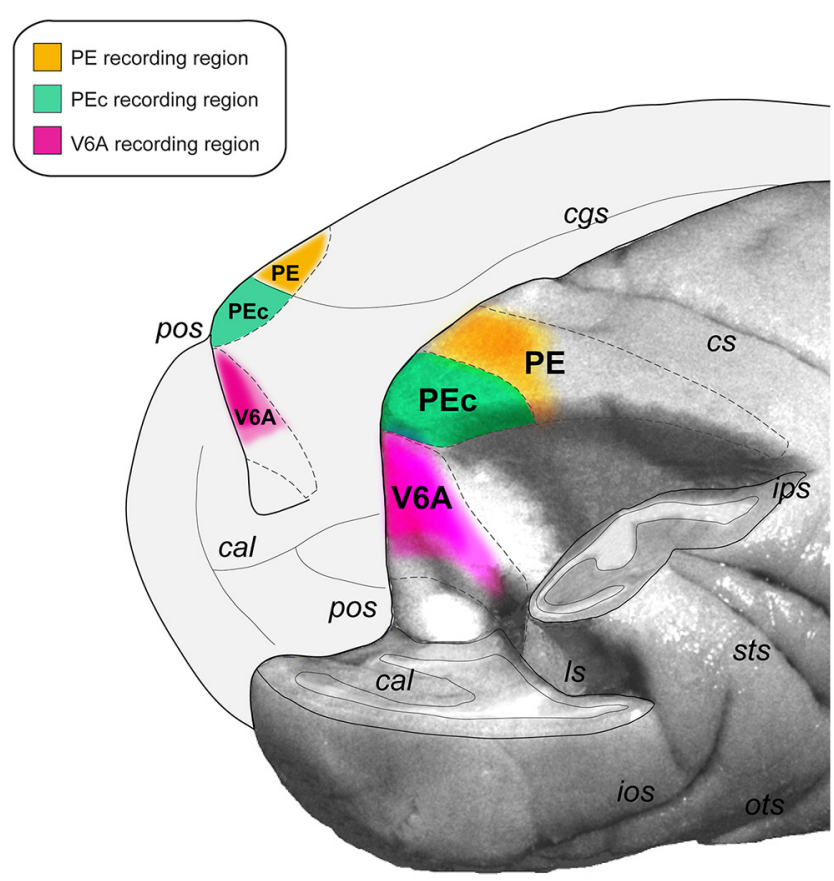

Figure 1. Areas of the superior parietal lobule in a macaque brain. Posterolateral view of a part of $M$. fascicularis brain showing location and extent of areas V6A, PEc, and PE (outlined by gray dashed lines) of the SPL. The right hemisphere is partially dissected to show the areas hidden in the parieto-occipital and intraparietal sulci. Colored areas represent the reconstructions of the recording regions within these areas as a mean of two animals and four hemispheres. cal, calcarine sulcus; cgs, cingulate sulcus; cs, central sulcus; ios, inferior occipital sulcus; ips, intraparietal sulcus; Is, lunate sulcus; ots, occipitotemporal sulcus; pos, parieto-occipital sulcus; sts, superior temporal sulcus (modified from Gamberini et al., 2020).

extended and motionless, and the hand was far from the body.

Stimuli presentation and animals' performance were monitored using custom software written in Labview (National Instruments), as described previously (Kutz et al., 2005); if monkey broke fixation, made an incorrect arm movement, or did not respect the temporal constraints of the task, the trial was aborted. Microswitches (monopolar microswitches, RS Components) were mounted under the HB and under each LED to monitor the correct performance of arm movements. Eye position signals were sampled with two cameras (one for each eye) of an infrared eye-tracking system (ISCAN) at $100 \mathrm{~Hz}$ and were controlled by an electronic window $\left(4^{\circ} \times 4^{\circ}\right)$ centered on the fixation target. If the monkey fixated outside this window, the trial was aborted. The task was performed in darkness, in blocks of 90 randomized trials, 10 for each LED target position.

At the beginning of each recording session, the monkey was required to perform a calibration task to calibrate the eye tracker. In this task, animal fixated 10 LEDs mounted on a frontal panel at $15 \mathrm{~cm}$ from the eyes. For each eye, signals to be used for calibration were extracted during the fixation of five LEDs, one central aligned with the eye's straight-ahead position, and four peripheral ones placed at an angle of $\pm 15^{\circ}$ (distance: $4 \mathrm{~cm}$ ) both in the horizontal and vertical axes. From the two individual calibrated eye position signals, we derived the mean of the two eyes (the conjugate or version signal), and the difference between the two eyes (the disconjugate or vergence signal) using the equations: Version $=(R+L) / 2$ and Vergence $=L-R$, where $R$ and $L$ were the position of the right and left eye, respectively, expressed in degrees. The version and vergence values were also used by the LabVIEW software to control the gaze position and abort trials in case of incorrectness.

\section{Surgical and recording procedures}

After training completion, a head-restraint system and a recording chamber were surgically implanted in asepsis and under general anesthesia (sodium thiopental, $8 \mathrm{mg} /$ $\mathrm{kg} / \mathrm{h}$, i.v.) following the procedures reported in Galletti et al. (1995). Adequate measures were taken to minimize pain or discomfort. A full program of postoperative analgesia (ketorolac trometazyn, $1 \mathrm{mg} / \mathrm{kg}$, i.m., immediately after surgery, and $1.6 \mathrm{mg} / \mathrm{kg}$, i.m., on the following days) and antibiotic care [Ritardomicina ${ }^{\circledR}$ (benzathine benzylpenicillin + dihydrostreptomycin + streptomycin) 1-1.5 $\mathrm{ml} / 10 \mathrm{~kg}$ every 5-6 d] followed the surgery.

Single-cell activity was extracellularly recorded from areas V6A, PEc, and PE of the two monkeys (Fig. 1). We performed single microelectrode penetrations using a 5channel multielectrode recording system (MiniMatrix, Thomas Recording, GmbH). The electrode signals were amplified (at a gain of 10,000 ) and bandpass filtered (between 0.5 and $5 \mathrm{kHz}$ ). Action potentials in each channel were isolated online with a waveform discriminator (Multi Spike Detector; Alpha Omega Engineering). Spikes were sampled at $100 \mathrm{kHz}$. The present study includes neurons assigned to areas V6A, PEc, and PE following the cytoarchitectonic criteria of Pandya and Seltzer (1982) and Luppino et al. (2005).

\section{Data analysis}

All the analyses were performed using custom scripts in MATLAB (MathWorks, RRID: SCR_001622). Analysis of the neuronal activity during the Fixation-to-Reach task was made by quantifying the discharge recorded during each trial in the following time epochs (Fig. 2):

- FIX: from $500 \mathrm{~ms}$ after fixation onset (corresponding to the onset of ocular fixation inside the electronic window) until $1000 \mathrm{~ms}$ after it. It contains the neural discharge for LED fixation, avoiding transient saccaderelated responses (see Kutz et al., 2003).

- HOLD: from 200 ms after LED pressing until $700 \mathrm{~ms}$ after it. It contains the discharge of the cells during hand holding, avoiding transient responses related to the stop of the arm movement.

We included in the analyses only those units recorded during at least seven trials per spatial position, and with a mean firing rate in HOLD and/or in FIX higher than three spikes/s in at least one position. The reasons for these conservative criteria are dictated by the intrinsic high 

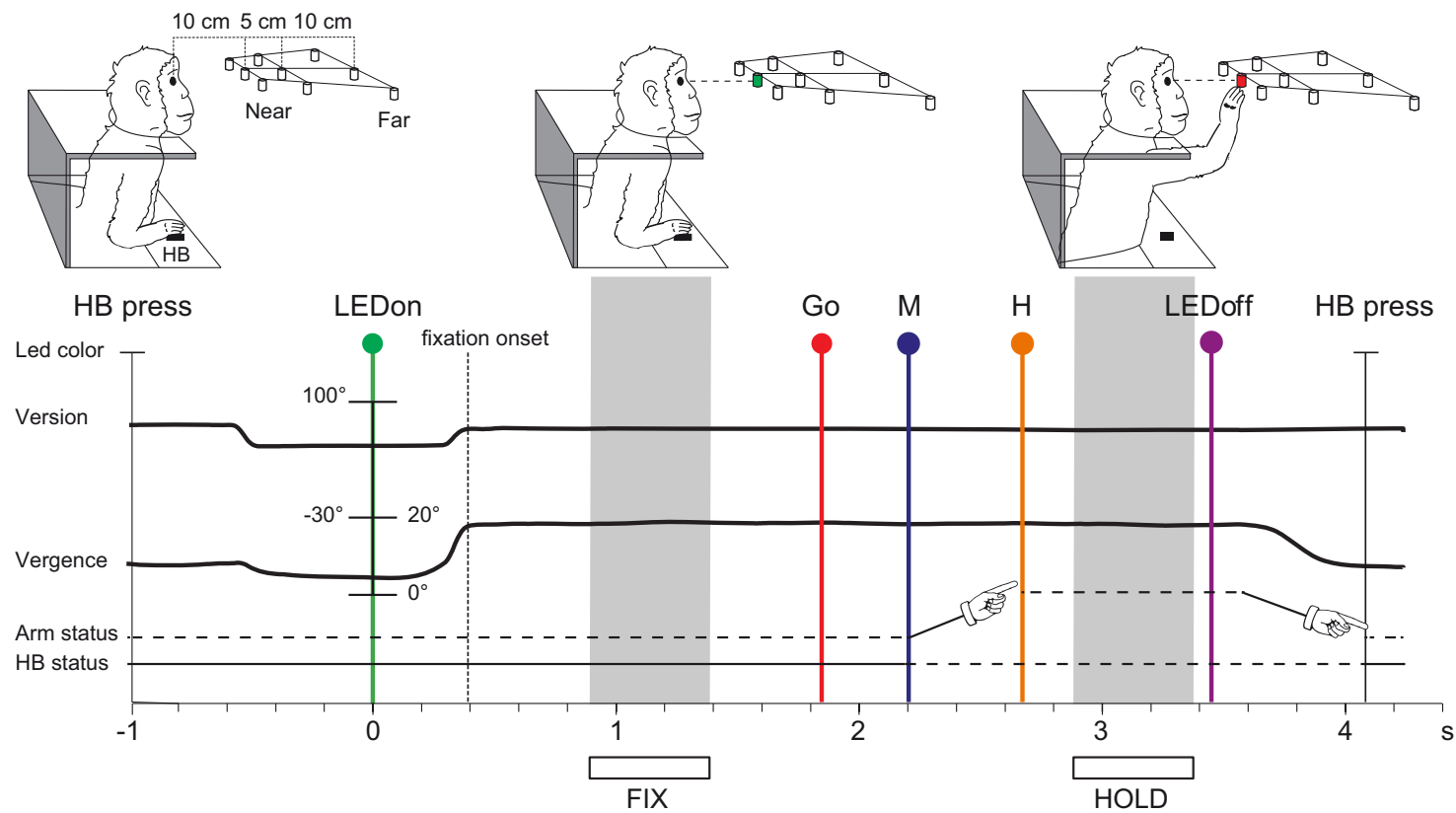

Figure 2. Scheme of the experimental setup and timing of the task. The monkey sat in a primate chair in front of a horizontal panel located at eye level with nine LEDs used both as fixation and reaching targets. HB, home button. The distances in depth between the three targets of the central row from mid-eye level are shown. The time sequence of task events shows LED status, the eye's vergence and version traces, arm status, and HB status. From left to right, vertical lines indicate, respectively, trial start (HB press, black line), target appearance (LEDon, green line), fixation onset (end of saccade movement, dashed line), go signal (Go, red line), arm movement onset ( $\mathrm{M}$, blue line), holding phase of the target $(\mathrm{H}$, orange line), turning off of the LED (LEDoff, purple line), and trial end (HB press, gray line). Arm drawings indicate the forward and backward arm movement. The relevant time intervals (epochs) used for the analysis of neural activity are indicated with gray areas and white bars below the time axis: FIX = fixation epoch, HOLD = holding epoch.

variability of biological responses in the PPC as explained in detail in Kutz et al. (2003).

To assess the effect of the eye and arm position on V6A, PEc, and PE cells, we performed a two-way ANOVA with factors being the epoch (two levels: FIX and HOLD) and target positions (nine levels: nine spatial positions of the reaching targets). FIX was chosen as a reference because in this epoch the gaze was still and the monkeys were not required to execute any arm movement. We defined as task-related and further analyzed those cells showing significant main effects of both target positions and epoch $(p<0.05)$, significant interaction (target positions ${ }^{*} e p o c h, p<0.05$ ), or a cumulative main and interaction effect (epoch +interaction, target positions + interaction).

Significant modulation of neural activity by the target position in each epoch of interest was assessed by a oneway ANOVA (factor: target position, $p<0.05$ ). The incidence of task-related cells with significant modulations by the target position during HOLD, FIX and both FIX\&HOLD epochs was compared in the three areas V6A, PEc, PE with a z-test (Zar, 1999), as detailed in Fluet et al. (2010). To perform this test, the SE of the sampling distribution difference between two proportions was computed as:

$$
S E=\sqrt{p(1-p)[(1 / n 1)(1 / n 2)]}
$$

with $p=[(n 1 \times p 1)(n 2 \times p 2)] /(n 1+n 2)$ representing the pooled sample proportion and $n 1 / p 1$ and $n 2 / p 2$ representing the size and proportion, respectively, of each sample. Subsequently, the $z$ score was calculated as $z=(p 1-p 2) / S E$, and its corresponding $p$ value was obtained from the (cumulative) normal distribution.

A z-test was also used to compare the incidence of task-related cells with higher firing rate during HOLD with respect to FIX in all the nine positions (referred as excited cells) and with lower firing rate during HOLD with respect to FIX in all the nine positions (referred as inhibited cells).

To analyze the spatial tuning of task-related cells activity during the time course of the task, a stepwise multiple linear regression model was applied with a sliding window approach (window-bin width: $250 \mathrm{~ms}$; step: $50 \mathrm{~ms}$ ). A similar method has been used in previous publications from our lab (Hadjidimitrakis et al., 2014a, 2015; De Vitis et al., 2019). To dynamically relate the neural activity to the different target positions over time, we applied the following equation for the firing rate using this regression model:

$$
A(X i, Y i)=b 0+b 1 X i+b 2 Y i
$$

where $A$ was the neural activity in spikes per second for the ith trials; $\mathrm{Xi}$ and $\mathrm{Yi}$ the positions of the target defined as vergence and version angles, respectively, of the eyes; b1 and b2 were regression coefficients and b0 the intercept. After being tested for their significance, the vergence and version coefficients were normalized with the standard deviation of vergence and version, correspondingly. In each bin, the sign of the significant linear correlation coefficients was used to determine the spatial preference per each neuron. 
Population responses of neurons modulated by the target position during HOLD and FIX\&HOLD epochs were computed as averaged spike density functions (SDFs). An SDF was calculated (Gaussian kernel, half-width $40 \mathrm{~ms}$ ) for each neuron included in the analysis and averaged across all the trials for each target position. The peak discharge of the neuron found over all the nine target positions during the epoch of interest (HOLD or FIX) was used to normalize all the SDFs. The normalized SDFs were then averaged to obtain population responses (Marzocchi et al., 2008). To statistically compare the population SDFs curves of best and worst positions in each area, we performed a permutation test (10,000 iterations), comparing the sum of squared errors of the actual and randomly permuted data. Comparisons of responses to target fixation have been made in the interval from 500 to $1000 \mathrm{~ms}$ after saccade offset for FIX. Comparisons of responses related to static positions of the arm have been made in the interval from $200 \mathrm{~ms}$ after the LED pressing until $700 \mathrm{~ms}$ after it for HOLD. The onset of spatial selectivity was calculated as the time of divergence of population SDFs of the best and worst target position (half-Gaussian kernel, width $5 \mathrm{~ms})$.

\section{Results}

We have investigated the influence of gaze and proprioceptive signals from the arm on the activity of neurons of three SPL areas (V6A, PEc, PE) in two macaque monkeys. Animals performed a Fixation-to-Reach task being instructed to fixate and reach nine foveated targets located at different spatial locations in the $3 \mathrm{D}$ space facing the animal (Fig. 2). Only the horizontal plane at eye level was explored to reduce the factors influencing neuronal activity, being well known that gaze elevation modulates the activity of neurons in the caudal part of SPL (Galletti et al., 1995; Breveglieri et al., 2012). The task allowed us to test the influence of gazing different positions of the peripersonal space (epoch FIX) and of holding the arm in different spatial configurations (epoch HOLD) on neuronal activity.

We recorded the activity of 303 single V6A cells (left hemisphere: 218, right hemisphere: 85; Monkey A: 168, Monkey B: 135), 264 PEc cells (left hemisphere: 159, right hemisphere: 105; Monkey A: 157, Monkey B: 107), and 189 PE cells (left hemisphere: 91, right hemisphere: 98; Monkey A: 69, Monkey B: 120). We analyzed neural responses during two epochs: target fixation (FIX, from 500 ms after fixation onset until $1000 \mathrm{~ms}$ after it; Fig. 2) and target holding (HOLD, from $200 \mathrm{~ms}$ after the LED pressing until $700 \mathrm{~ms}$ after it; Fig. 2).

\section{Effect of eye and arm position signals on V6A, PEc, and PE}

We examined how many V6A, PEc, and PE neurons were significantly modulated by the eye and arm position (two-way ANOVA, $p<0.05$ ) and identified them as "taskrelated". A total of $226 / 303$ V6A cells $(75 \%), 188 / 264$ PEc cells $(71 \%)$, and $85 / 189$ PE cells $(45 \%)$ showed task-related activity and were further analyzed.

Figures 3-5 show three examples of neurons modulated during the Fixation-to-Reach task, recorded from the areas V6A, PEc, and PE, respectively. Task-related V6A neuron showed in Figure 3 was modulated by the spatial position of reaching target both in FIX (one-way ANOVA, $p=3 \times 10^{-5}$ ) and HOLD (one-way ANOVA, $p=3 \times 10^{-8}$ ) and showed a higher discharge during HOLD for far targets, mainly the ipsilateral one. This cell displayed low activity during arm movement and peaked in discharge after the target LED pressing, when the monkey's arm was still and extended (HOLD). Task-related PEc neuron showed in Figure 4 exhibited a spatial preference for positions ipsilateral and near the body (one-way ANOVA, $p=0.001$ during FIX, $p=10^{-6}$ during HOLD). Cell's activity gradually increased after the go signal, peaked around the target LED pressing and decreased afterward, but remained quite high during the HOLD epoch. In contrast to V6A, PE neuron discharged strongly during arm movement (Fig. 5). Its activity was modulated by the spatial position of the arm during HOLD (one-way ANOVA, $p=5 \times 10^{-5}$ ), whereas the activity during FIX was comparable in the nine target positions $(p=0.05)$.

Different functional features were observed in PE on one side and in PEc/V6A on the other: firstly, a lower incidence of task-related cells was found in PE compared with both PEc and V6A (z-test, PE vs PEc $p=2 \times 10^{-8}$, PE vs V6A $p=5 \times 10^{-6}$ ), whereas no significant difference was found between V6A and PEc (z-test, $p=0.4$ ). We categorized neurons in four classes, according to their modulation by fixation and arm holding in space (epoch, EPO in Fig. $6 A$ ) and by the nine spatial positions (target positions, POS in Fig. $6 A$ ) during one or both the epochs of interest. Therefore, we distinguished: (1) neurons significantly modulated by the eye position, the arm position in space, or by both (POS+EPO; Fig. 6A); (2) neurons modulated only by the interaction POS*EPO (INT; Fig. $6 A$ ); (3) neurons modulated by INT and either the nine spatial positions (INT+POS; Fig. 6A) or (4) the epochs of interest (INT+EPO; Fig. 6A). Comparing the results in the three areas, we have found no statistical differences between the four categories of task-related cells in both V6A and PEc, whereas some dissimilarities appeared between the two visuomotor areas and PE: compared with PE, V6A and $\mathrm{PEC}$ contain a higher percentage of neurons modulated by eye position, arm position or both (POS+EPO, V6A 52\%, PEc 48\%, PE 28\%; see Fig. $6 A$ ) and a higher incidence of neurons modulated by the interaction factor and target positions (INT+POS, V6A 16\%, PEc 14\%, PE 4\%; see Fig. $6 A$; z-test, V6A vs PE, POS +EPO $p=1 \times$ $10^{-7}$, INT+POS, $p=0.0001 ;$ PEc vs PE, POS +EPO $p=10^{-5}$, INT+POS, $p=0.0009$; V6A vs PEc POS +EPO $p=0.3$, INT +POS, $p=0.5$ ). Also, the proportion of cells modulated by the interaction factor and epochs (INT+EPO) was similar in V6A and PEc (z-test, V6A vs PEc $p=0.1)$ with a lower incidence of these cells in V6A compared with PE (z-test, V6A vs PE, $p=0.01$ ) and no significant difference between $P E c$ and PE (ztest, PEc vs PE $p=0.2$; V6A 4\%, PEc 7\%, PE 10\%; see Fig. $6 A$ ). Cells modulated only by the interaction were similarly represented in all the three areas (V6A 3\%, PEc 3\%, PE 3\%; see Fig. 6A; z-test, $p>0.05$ for all comparisons). 

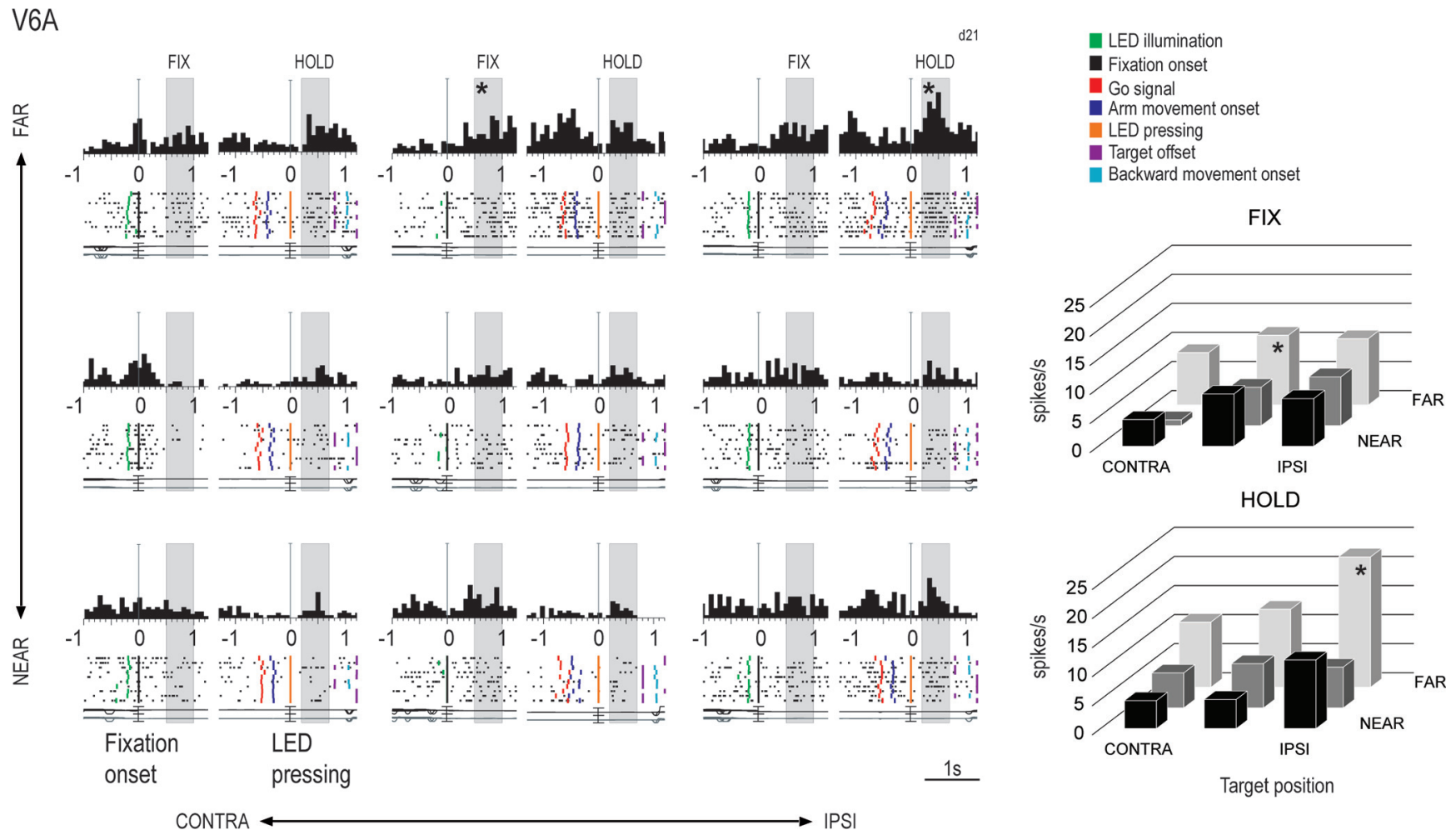

Figure 3. Example V6A neuron tuned by eye/arm positions both in FIX and HOLD. Left, Spike histograms (top), rasters (middle), eye traces (bottom) are shown for each of the nine target positions tested. Target positions were arranged in three directions (columns; contralateral, central, ipsilateral with respect to the recording hemisphere) and three depths (rows; far, intermediate, near with respect to the monkey's body). Colored vertical lines along rasters indicate behavioral markers that, from left to right, are: LED illumination, fixation onset, go signal, movement onset, movement end (LED pressing), target offset, backward movement onset. Thin vertical lines along spike histograms indicate the alignment of activity at the fixation onset and LED pressing, respectively. Realignment is evidenced with a gap in histograms. Epochs of interest are represented within gray rectangles. Vertical scale on histograms: 40 spikes/s. Right, Distribution of the mean activity of the same cell across trials during epochs FIX (top) and HOLD (bottom) for each of the nine target positions tested. Asterisks indicate the spatial position evoking the highest discharge in each epoch.

\section{A reverse trend of eye-hand position tuning in $\mathrm{V} 6 \mathrm{~A} /$ PEc and PE}

To quantify the proportion of cells tuned by the target positions in FIX, HOLD, and in both epochs, we performed a one-way ANOVA $(p<0.05)$. Figure $6 B$ shows the incidence of task-related cells tuned by eye positions (FIX) and/or arm positions in space (HOLD) separately for each area. We found that the distribution of V6A, PEc, and PE cells based on their tuning for reaching targets was different across task epochs (Fig. 6B). Cells modulated only during FIX represented almost $30 \%$ of the total cell population in all three areas (z-test, $p>0.05$ for all comparisons). Instead, cells modulated in HOLD and in both FIX and HOLD showed a clear and opposite trend from V6A to PE: cells modulated only by the position of the arm (HOLD) increased going from V6A to PE (z-test, V6A vs PEc, $p=4 \times$ $10^{-7}$; V6A vs PE, $p=7 \times 10^{-8}$ ), whereas cells modulated by eye-position and arm-position (FIX\&HOLD) progressively decreased from V6A to PE (z-test, V6A vs PEc, $p=0.0008$; V6A vs PE, $p=8 \times 10^{-9}$; PEc vs PE, $p=0.002$ ).

Moreover, within the same area we found some dissimilarities in the categories of cells spatially tuned in V6A and PEc, but not in PE. In V6A and PEc, cells spatially modulated during both FIX and HOLD were more represented
(64\% and $47 \%$ respectively) than those cells tuned only in one epoch (z-test, $p<0.01$ for all comparisons), supporting the view that these areas are more implicated in eyehand coordination, being highly sensitive to both the direction of gaze and to the arm proprioceptive signals. Furthermore, in V6A we observed a lower incidence of neurons modulated only during HOLD with respect to those modulated only during FIX (z-test, $p=3 \times 10^{-6}$ ), and this is in line with the increase of somatosensory and the simultaneous decrease of visual processing observed along the caudo-rostral axis of the medial SPL. In PE we did not find any statistical differences in the subgroups of cells ( $z$-test, $p>0.05$ for all comparisons).

To sum up, these results show the existence of a decreasing trend for cells spatially modulated during both fixation and arm holding going from V6A to PEc and then to PE. This coding scheme parallels the gradual shift from joint to separate processing of amplitude and directions signals of arm movement during reaching (Hadjidimitrakis et al., 2014a, 2015; De Vitis et al., 2019).

\section{Dynamic space representation along the task}

To characterize the spatial preference of task-related neurons during the time course of the task, a sliding 
PEC
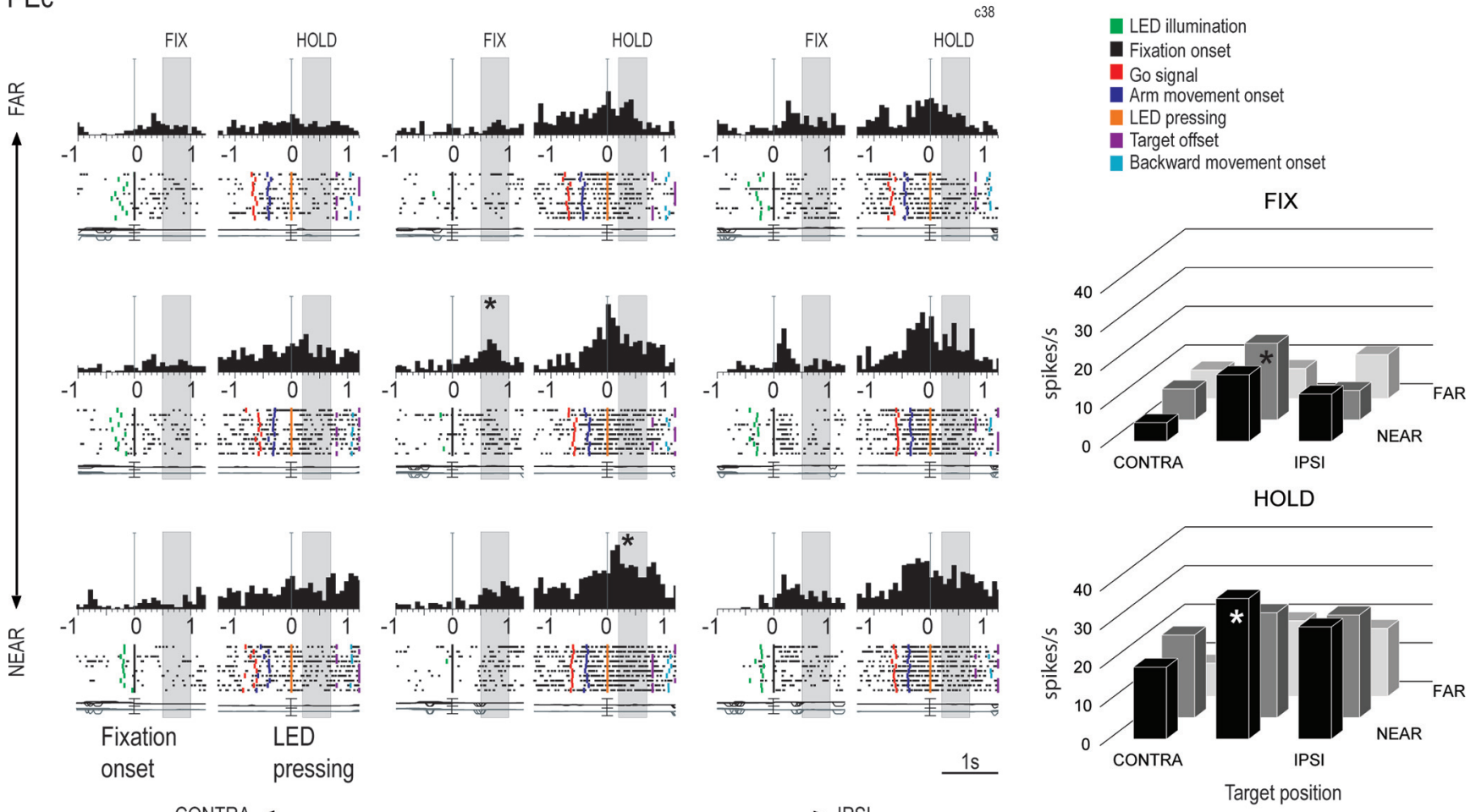

Figure 4. Example PEc neuron tuned by eye/arm positions both in FIX and HOLD. All the conventions as in Figure 3. Vertical scale on histograms: 70 spikes/s.

PE

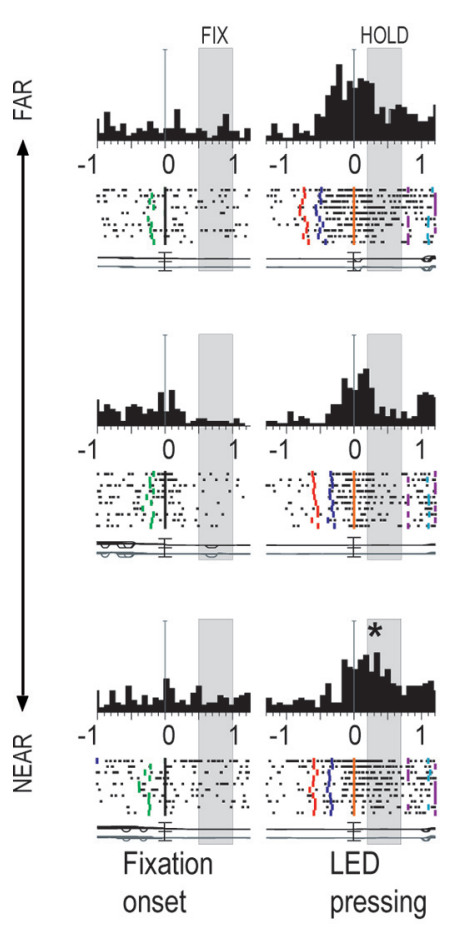

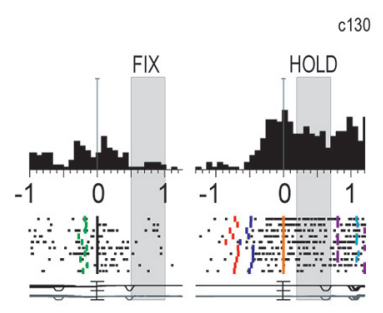
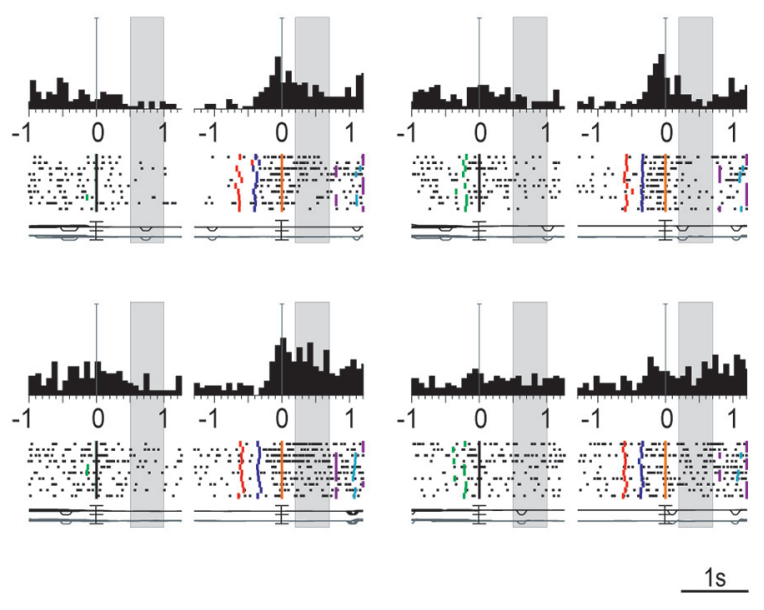
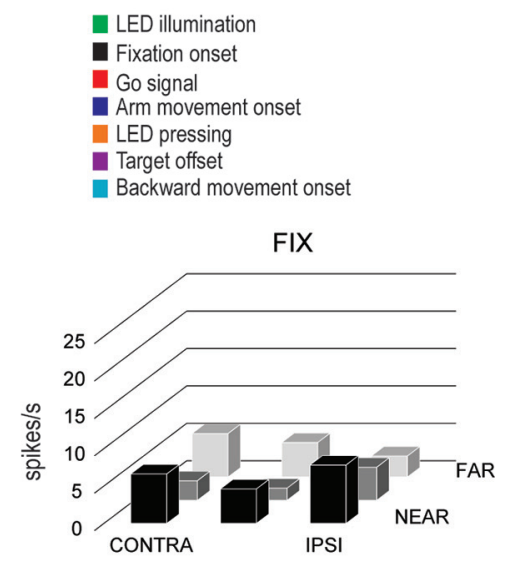

FIX

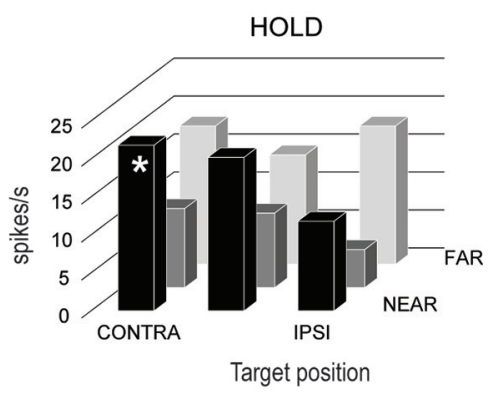

Figure 5. Example PE neuron tuned by eye/arm positions only in HOLD. All the conventions as in Figure 3 . Vertical scale on histograms: 48 spikes/s. 
A Task related neurons

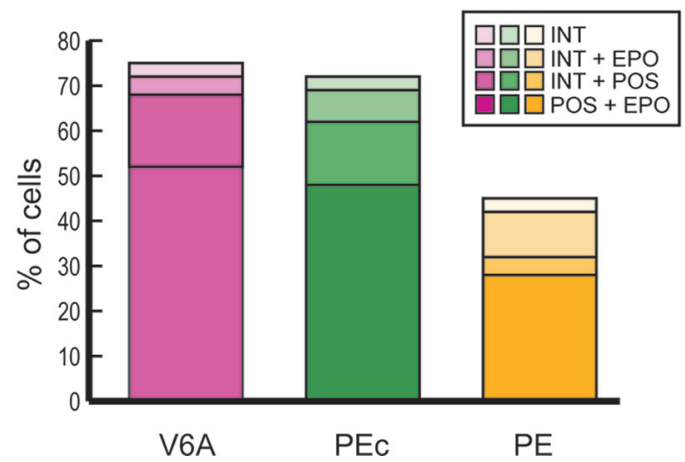

B

Tuning for reaching target

positions

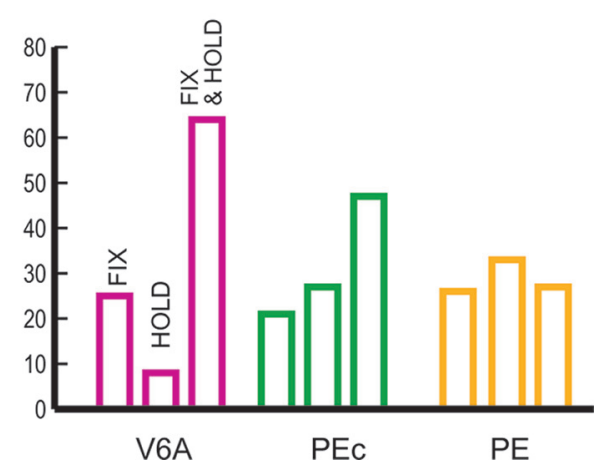

Figure 6. Significant effects modulating V6A, PEc, and PE cells. $A$, The histograms show the results of a two-way ANOVA as the incidence of cells modulated by the fixation and arm holding in space (EPO: epoch) and the nine spatial positions in one or both the epochs of interest (POS: target positions), or by the interaction of the two factors (INT: target positions*epoch). Numbers of modulated cells for each subgroup of task-related cells: POS+EPO, V6A N=157, PEc =126, PE = 52; INT+EPO, V6A $N=12, \mathrm{PEC}=18$, $\mathrm{PE}=19$; INT+POS, V6A $N=47, \mathrm{PEC}=36, \mathrm{PE}=8 ; \mathrm{INT}, \mathrm{V} 6 \mathrm{~A} N=10, \mathrm{PEc}=8, \mathrm{PE}=6$ (cells with no effect, $\mathrm{V} 6 \mathrm{~A} N=27, \mathrm{PEC}=22$, $\mathrm{PE}=36$ not shown in figure). $\boldsymbol{B}$, Percentages of cells tuned by the position of the reaching target in FIX, HOLD, and FIX\&HOLD, as a result of a one-way ANOVA.

window linear regression analysis was performed, considering target depth and direction as independent variables. Neurons with a significant linear vergence tuning were classified as NEAR or FAR, whereas cells linearly tuned by version angle were classified as CONTRA or IPSI, depending on both the sign of the correlation coefficient and the recording hemisphere. The percentage of V6A, PEc, and PE cells falling into the above groups is illustrated in Figure 7. Regarding the neuronal preference for depth, V6A neurons equally represented NEAR and FAR reachable space during the time course of the task, with a slight preference for farther positions at the end of the holding phase (Fig. 7, left, two-sample Kolmogorov-Smirnov test, $p<0.01)$. PEc cells showed instead a stronger tuning for FAR space from the beginning of the trial (FIX, twosample Kolmogorov-Smirnov test, $p<0.01$ ), and this predominance was maintained until movement execution, after which, in the HOLD phase, the proportion of neurons preferring FAR positions matched that of neurons preferring NEAR positions (Fig. 7, left, two-sample Kolmogorov-Smirnov test, $p>0.05)$. PE neurons tuned for FAR reachable space were found to be more represented than those tuned for NEAR space during the course of the whole trial (Fig. 7, left, two-sample KolmogorovSmirnov test, $p<0.01$ in FIX and HOLD). This remarkable preference for FAR space likely reflects the strong influence of somatosensory input in PE. When the monkey reaches the farthest positions, the arm hyperextends to touch the target, and this leads to a strong somatosensory stimulation evoked by shoulder, elbow and wrist rotation. Regarding the directional tuning (Fig. 7, right), IPSI neurons were more numerous than CONTRA ones in V6A, particularly during target fixation and holding (two-sample Kolmogorov-Smirnov test, $p<0.01$ ), whereas these two categories of neurons were equally represented in PEc (two-sample KolmogorovSmirnov test, $p>0.05$ in FIX and HOLD). In turn, PE cells showed a gradual shift from a slight preference for the
CONTRA space in the early part of the trial (two-sample Kolmogorov-Smirnov test, $p<0.05$ in FIX) to a more pronounced preference for the IPSI space in HOLD (Fig. 7, right, two-sample Kolmogorov-Smirnov test, $p<0.01$ ).

We then investigated how constant the preference for a given position (i.e., NEAR vs FAR or IPSI vs CONTRA) during the time course of the task was. To evaluate the consistency of spatial preference across single neurons, we quantified the cells that retained, altered, lost, or acquired their spatial preference in couples of subsequent bins of $50 \mathrm{~ms}$. The overall tendency of cells from all the three areas was to retain their spatial preference both in depth and direction (i.e., vergence and version). Cells that did not alter their preference as the task progressed were the most represented in all the three areas $(50-60 \%$ in V6A, $40-50 \%$ in PEc, $30-40 \%$ in PE), from fixation onset until target pressing, both in depth and direction. The proportion of cells preserving their depth tuning slightly decreased during target holding (epoch HOLD) in all three areas $(40 \%$ in $\mathrm{V} 6 \mathrm{~A}, 30-40 \%$ in PEc, $20-30 \%$ in PE). Only a minority of cells from all the three areas (10-15\%) lost or acquired their tuning, and very few cells $(<3 \%)$ changed their spatial preference during the time course of the trial, both in depth and direction. In summary, the trends were similar for all the three areas both in depth and direction, with a remarkably stable proportion of spatially tuned cells that retained their tuning as the trial progressed, from target fixation to LED pressing.

\section{Population responses}

The timing of neural activity during the task and the spatial tuning of cells modulated by eye-position and/or armposition is evident from the population SDFs (see Materials and Methods) shown in Figure 8, where neural activity was ranked according to each cell's preference in FIX (Fig. $8 A$ ) and HOLD (Fig. 8B). In Figure $8 A-B$, cell 


\section{Space representation in the SPL}

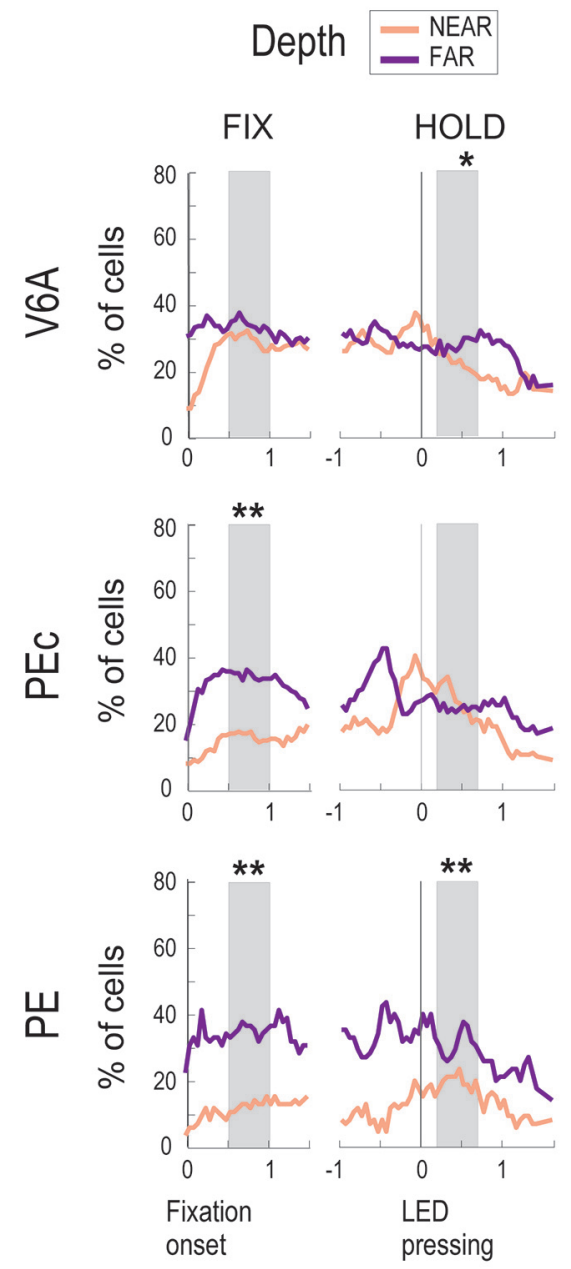

Direction $=$ IPSI
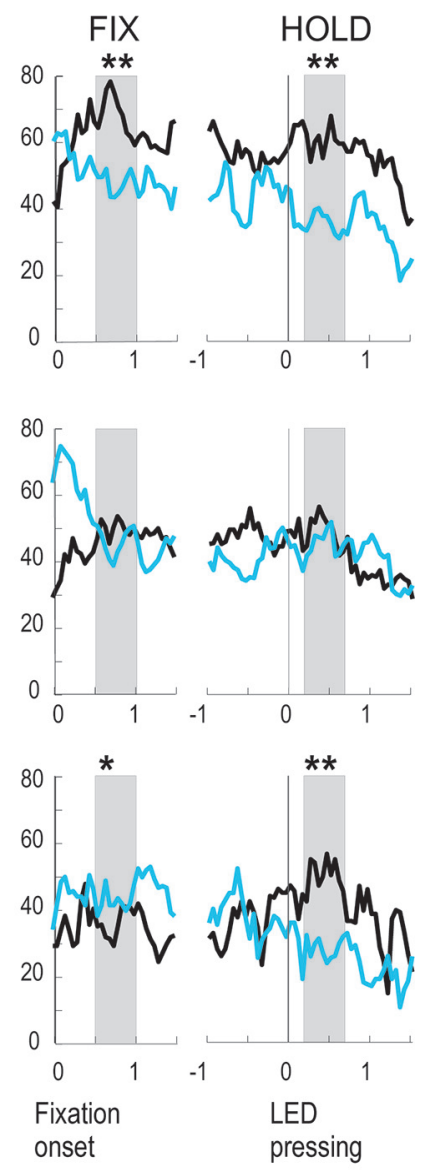

Figure 7. Space representation in the three SPL areas along the task. Percentage of V6A (top), PEc (middle), and PE (bottom) taskrelated cells linearly modulated by depth (left) and direction (right) showing a preference for far (FAR, purple line) or near (NEAR, pink line) space and ipsilateral (IPSI, black line) or contralateral (CONTRA, light blue line) space with respect to the recording hemisphere in a sliding window linear regression (window-bin width: $250 \mathrm{~ms}$; step: $50 \mathrm{~ms}$ ). Asterisks indicate significant differences between curves in bins of $250 \mathrm{~ms}$ (two-sample Kolmogorov-Smirnov test, $p<0.01$ ). Other conventions as in Figure 3.

responses were classified from the strongest one, elicited by a certain target position for a given neuron (whatever the position of the target was), hereafter called BEST, the second best response, the third, the fourth, and so on, up to the ninth, corresponding to the weakest response for the same neuron, hereafter called WORST, during FIX and HOLD epochs, respectively.

After ranking the neural activities according to the spatial preferences during FIX, we found that the activity during fixation started to diverge around the fixation onset in V6A and PEc (Fig. 8A, top and middle panels, permutation test, best vs worst curves, V6A $p=0.003$, PEc $p=0.008$ ), while in $\mathrm{PE}$ neural responses diverged later on, after the fixation onset (Fig. 8A, bottom panels, permutation test, PE $p=0.004)$. The size of the tuning was similar in the three areas in FIX, but the modulation lasted longer in V6A and PEc than in PE (compare the significance bars reported in each panel).

After ranking the neural activities according to spatial preferences during HOLD, we still found a tuning effect during FIX in V6A (Fig. 8B, top panels), where BEST and WORST position lines started to diverge $40 \mathrm{~ms}$ after the fixation onset and remained well separated for the remaining part of the trial (permutation test, best vs worst curves, V6A $p=0.007$ ). During HOLD, the activity in V6A was strongly tuned: the curves for all nine conditions appeared to be unraveled and well distinct, with activities for the BEST and second best conditions being continuously higher than the baseline (FIX activity), and the ones for conditions seventh, eighth, and WORST being progressively more inhibited than the activity during FIX. In PEc (Fig. 8B, middle panels), the modulation during FIX, although 
A Activity ranking based on FIX
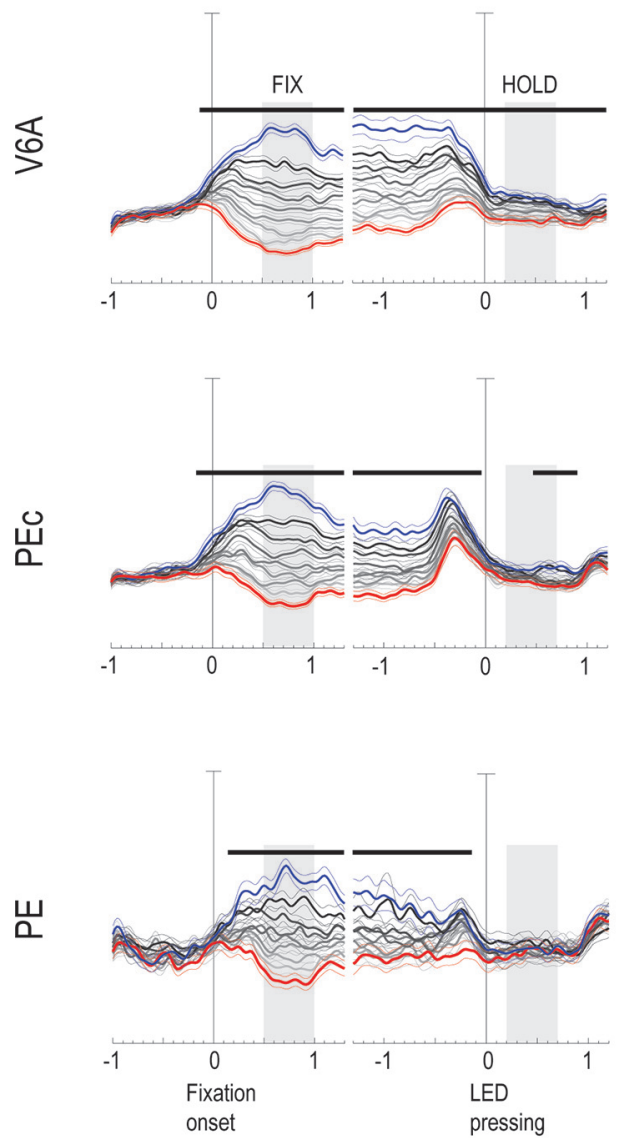

B Activity ranking based on HOLD
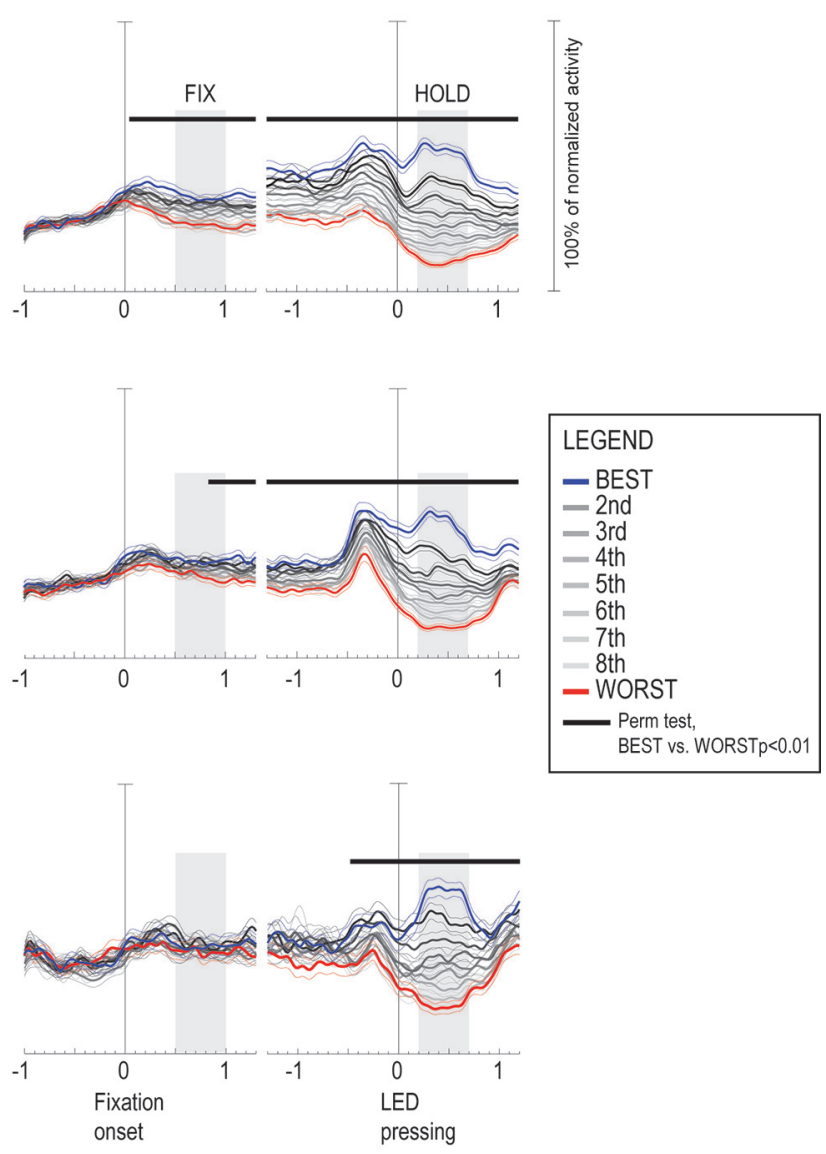

Figure 8. Population activity. Population activity of V6A (top), PEc (middle), and PE (bottom) cells modulated by the position of reaching target during FIX and/or HOLD, expressed as averaged normalized SDFs (thick lines) with variability bands (SEM; thin lines). $\boldsymbol{A}$, SDFs obtained by ranking the activity of each neuron according to the intensity of the response for each spatial position elicited in FIX for that neuron. $\boldsymbol{B}$, SDFs obtained ranking the activity of each neuron according to the intensity of the response for each spatial position elicited in HOLD. Neuronal activities have been aligned twice at the onset of fixation and at LED pressing. Vertical bars in all SDF plots: $100 \%$ of normalized activity. Permutation test was performed on BEST (blue line) and WORST (red line) curves in all the time intervals shown. At the top of each panel, black bars are used to indicate the significancy (see legend).

significant (permutation test, best vs worst curves, PEc $p=0.008$ ), was much weaker than in V6A, and in PE (Fig. $8 B$, bottom panels) it was completely absent $(p>0.05)$. During HOLD, the same strong tuning was present in PEC and $\mathrm{PE}$, and it looked similar in the three areas. It is also evident, though not indicated by a specific epoch in Figure 8, that the activity during the execution of arm movement (the period just before the alignment to the LED pressing) was strongly tuned in V6A and PEc and weaker in PE.

Interestingly, while in V6A and, although to a lesser extent, PEc, the spatial tuning was evident during both FIX and HOLD, regardless of the epoch on which the ranking was based (Fig. $8 A$ and $8 B$ ), in PE the modulations were evident only if the ranking was made according to the given epoch (i.e., clear tuning in FIX and no modulation in HOLD if the ranking was based on the activity in FIX, Fig. $8 A$; the opposite trend if the ranking was based on the activity in HOLD, Fig. $8 B$ ). This suggests that the ranking orders in PE were different during fixation and arm holding, and that the neural representations of these two signals (eye position, proprioception) in PE are independent. To sum up, V6A and PEc neurons showed similar temporal evolution in both FIX and HOLD, being their activity jointly influenced by eye position and arm movement-related information, whereas PE cells seemed to be more involved in the encoding of proprioceptive signals from the arm rather than oculomotor signals.

As evident from the population data, the activity for the WORST position in HOLD is lower than in FIX. This suggests that cells could be further inhibited by the position of the arm, in addition to the inhibition because of eye position. So, we calculated the incidence of task-related cells excited (i.e., with a higher firing rate) or inhibited (i.e., with a lower firing rate) during HOLD with respect to the baseline activity (FIX) in each target position. As expected, we found more cells inhibited than cells excited in all the 
three SPL areas we tested: 33 cells inhibited (15\%) and 9 excited $(4 \%)$ in V6A (z-test, V6A $p=0.0001)$, 25 cells inhibited (13\%) and 10 excited (5\%) in PEc (z-test, PEc $p=0.01)$ and 12 cells inhibited (14\%) and 4 excited $(5 \%)$ in PE (z-test, PE $p=0.04)$. The incidence of inhibition was similar among the three areas.

\section{Discussion}

In this study, we investigated the modulating effect of eye-position and arm-position on neuronal activity in areas V6A, PEc, and PE while the animals performed a foveal reaching task. It has been recently suggested that V6A and PEc belong to the same cytoarchitectural sector of the SPL (Brodmann's area 7), whereas PE does not (Brodmann's area 5; Gamberini et al., 2020). Present data support this view showing similarities between the functional properties of V6A and PEc and differences with respect to PE. We have found that the incidence of taskrelated cells was lower in PE than in V6A and PEc $(75 \%$ in V6A; $71 \%$ in PEc; $45 \%$ in PE) and showed that V6A and PEc neurons exhibited similar spatial patterns of neural modulation during fixation and target holding, according to the target position. In contrast, in PE the neural representations during fixation and target holding were not joined, suggesting an independent encoding of eye and arm position. A similar trend has been observed in the neural processing of amplitude and direction of arm movement during reaching: in PE the neural substrates related to amplitude and direction were different (Lacquaniti et al., 1995; De Vitis et al., 2019), while in V6A a common neural substrate was observed (Hadjidimitrakis et al., 2014a). Our results revealed a decreasing trend for cells spatially modulated during both fixation and arm holding going from V6A to PEc, to PE (Fig. $6 B)$. This suggests that V6A and PEc may act as a bridge between pure visual cortices of the occipital lobe and rostral areas of the SPL more specifically involved in the proprioceptive control of action, like PE. This result is in line with the high sensitivity of both V6A and PEc to the direction of gaze, which is often a relevant cue to encode spatial coordinates of reaching targets (Galletti et al., 1995; Battaglia-Mayer et al., 2001; Ferraina et al., 2001; Raffi et al., 2008; Breveglieri et al., 2012; Hadjidimitrakis et al., 2014b), and to proprioceptive signals, particularly from the limbs (Breveglieri et al., 2002, 2008; Gamberini et al., 2018). The higher proportion of cells activated by a mix of gaze and arm signals in V6A and PEc than in PE (Fig. 6B) supports the view that these areas are more implicated in eye-hand coordination (Battaglia-Mayer et al., 2001; Ferraina et al., 2001; Diomedi et al., 2020).

The second trend emerging from our data is related to the incidence of cells modulated only during HOLD, which is much lower in V6A compared with PEc and PE. This result is consistent with the increasing percentage of neurons sensitive to somatosensory signals passing from V6A ( 30\%; Breveglieri et al., 2002), to PEc ( $65 \%$; Breveglieri et al., 2006, 2008; Gamberini et al., 2018), to PE (>90\%; Sakata et al., 1973; Mountcastle et al., 1975; De Vitis et al., 2019). In line with this view, it has been reported a predominance of eye-centered and mixed (eye/ hand-centered) cells in V6A (Bosco et al., 2016), and a prevalent employment of hand-centered reference frame in PEc (Hadjidimitrakis et al., 2014b; Piserchia et al., 2017) and PE (Lacquaniti et al., 1995).

\section{Role of PE in encoding arm posture}

While in the motor cortex the maintenance of a steady position of the arm is more related to the patterns of muscular contraction rather than to the posture per se (Evarts, 1969; Cheney and Fetz, 1980; Fromm, 1983), the sensitivity of the majority of Brodmann's area 5 cells to passive movements of the limbs (Sakata et al., 1973; Mountcastle et al., 1975) and to static arm positions (Georgopoulos et al., 1984; Kalaska and Hyde, 1985; Hamel-Pâquet et al., 2006; Cui and Andersen, 2011; McGuire and Sabes, 2011; Shi et al., 2013; De Vitis et al., 2019) has suggested a major role of this parietal area in encoding arm posture, pursuant to the current results regarding area PE. Despite the impact of the above cited papers about PE functional properties, only a few studies addressed the relative contribution of gaze direction and static hand positions signals in PE (Ferraina et al., 2009; De Vitis et al., 2019). Ferraina and colleagues' results highlighted the effect of both eye and hand information on PE neuronal activity, with a prevalence of hand information. Conversely, our results suggest a similar encoding of eye and arm signals in PE. The discrepancy could be because of the different experimental conditions, being the task used by Ferraina and colleagues a non-foveated reaching task, where armtarget positions changed while the coordinates of fixation-target remained constant. Another explanation could be the difference in the recording sites, since they studied a lateral sector of area PE, that only partially overlapped with our recording region (compare the yellow area in Fig. 1 with Fig. $1 b$ of De Vitis et al., 2019). Moreover, the data shown in Figures 6B, 8 suggest that an independent encoding of ocular and hand signals occurs in PE. This is in agreement with the independent encoding of version and vergence signals operated by PE neurons found by Lacquaniti (1995) and suggests that PE performs more specialized analysis of sensory signals than the regions located more caudally in the SPL, like PEc and V6A. Furthermore, we have found that a large proportion of PE cells tuned by depth showed a bias for FAR reachable space (Fig. 7), which most likely reflects a movement amplitude, corroborating the view that PE is involved in processing somatosensory and proprioceptive signals from the arm. Postural adjustments could be more important when the monkey reaches and holds the farthest targets and the integration of somatosensory and proprioceptive inputs from the arm could be reflected in increased levels of neural activity.

\section{Possible influence of spatial attention shifts on V6A activity}

Given the foveal nature of the reaching movements in our task, we cannot exclude that the responses observed during static arm positions could also reflect the overt spatial attention directed to the target besides the gaze (eye position) and the proprioceptive cues (arm position). 
In addition, covert shifts of spatial attention, during which the attentional focus is decoupled from gaze, which allows to direct the attention to a peripheral location without moving the eyes, may have contributed to modulate V6A neural activity. Several monkey and human experiments have revealed a crucial role of SPL during both overt and covert spatial attention shifts (Vandenberghe et al., 2001; Yantis et al., 2002; Molenberghs et al., 2007; Kelley et al., 2008; Galletti et al., 2010; Ciavarro et al., 2013; Caspari et al., 2015, 2018; Arsenault et al., 2018). In 2010, Galletti and colleagues showed for the first time attention-related activity in V6A at single cell level using a task where the monkey was required to covertly shift its attention from a central fixation point toward a peripheral location, and vice versa (Galletti et al., 2010). They found that the neural modulation was still present when attention was covertly shifted outward, to a peripheral cue, and demonstrated that visual, motor, and attentional responses can occur in combination in single V6A neurons. More recently, Caspari and colleagues (Caspari et al., 2015) have identified in monkeys a network of areas, including parietal area V6A, activated during spatial shifting events, using a spatial attention task adapted from a human fMRI study (Molenberghs et al., 2007). These findings could explain the proportion of V6A cells that we found to be inhibited during HOLD, possibly because of the modulating effect of the spotlight of attention. During HOLD, the spatial attention is likely to be covertly shifted out of the reaching target because the animal at that time has a more attractive object to attend -the HB that must be reached by its hand soon after the HOLD period to receive a reward.

We observed a similar percentage of cells inhibited during the target holding in all the SPL areas studied $15 \%$ in V6A, $13 \%$ in PEc, $14 \%$ in PE). Inhibited cells were also found by Gardner et al. (2007) in PE (41\%) and AIP (38\%) during the holding period after a grasping movement in a reach-to-grasp task. The inhibition was particularly relevant near the end of the holding period, just before the start of the backward arm movement to reach the initial hand position. Even in this case, spatial attention shifts (from positions on the panel, the reaching targets, to a position near the trunk, the $\mathrm{HB}$ ) could explain the observed results.

\section{SPL lesions impair visuomotor coordination during reaching}

The SPL sectors studied here are often damaged in patients affected by optic ataxia, a visuomotor coordination deficit that strongly impairs reaching actions (Rossetti et al., 2019). Very recent studies on optic ataxia patients performing reaching actions relying exclusively on proprioception showed that SPL lesions cause larger position errors than in healthy controls (Bartolo et al., 2018; Mikula et al., 2021). These studies highlight the crucial role of these regions in using proprioceptive information about hand position to correctly direct reaching movements (Mikula et al., 2021). Present data on single cells recording from monkey SPL may be the neurophysiological counterpart of this finding, with a deeper understanding of the stronger role of PE in estimating hand position basing on proprioceptive information and of
V6A and PEc in linking this input with gaze-related signals as well as visual inputs. All these areas are well equipped to contribute to the state estimation about upper limb status for controlling the correct execution of reaching movements (Fattori et al., 2017).

In conclusion, present data show that eye-position and arm-position modulation of neuronal activity is similar in areas V6A and PEc, and different in area PE. These results agree well with the recent suggestion that both $\mathrm{V} 6 \mathrm{~A}$ and PEc belong to Brodmann's area 7 while PE to Brodmann's area 5 (Gamberini et al., 2020). According to this view, we found that all three SPL areas integrate eye and limb position signals during the hand holding at the end of a foveal reaching, but the influence of the two signals is different in areas V6A and PEc with respect to PE. Area PE was found to be more sensitive to limb proprioceptive input while PEC and V6A, particularly this latter, were also influenced by the direction of gaze. These data support the existence, often reported in literature (Piserchia et al., 2017; Gamberini et al., 2018; De Vitis et al., 2019; Impieri et al., 2019), of a functional trend in the SPL, with the anterior part more involved in limb representation and the posterior one showing visuomotor characteristics well suited to control goal-directed actions.

\section{Conclusion}

Present data show that eye-position and arm-position modulation of neuronal activity is similar in areas V6A and PEc, and different in area PE. These results agree well with the recent suggestion that both $\mathrm{V} 6 \mathrm{~A}$ and $\mathrm{PEc}$ belong to Brodmann's area 7 while PE to Brodmann's area 5 (Gamberini et al., 2020). According to this view, we found that all three SPL areas integrate eye and limb position signals during the hand holding at the end of a foveal reaching, but the influence of the two signals is different in areas V6A and PEc with respect to PE. Area PE was found to be more sensitive to limb proprioceptive input while PEc and V6A, particularly this latter, were also influenced by the direction of gaze. These data support the existence, often reported in literature (Piserchia et al., 2017; Gamberini et al., 2018; De Vitis et al., 2019; Ilmpieri et al., 2019), of a functional trend in the SPL, with the anterior part more involved in limb representation and the posterior one showing visuomotor characteristics well suited to control goal-directed actions.

\section{References}

Arsenault JT, Caspari N, Vandenberghe R, Vanduffel W (2018) Attention shifts recruit the monkey default ode network. J Neurosci 38:1202-1217.

Bartolo A, Rossetti Y, Revol P, Urquizar C, Pisella L, Coello Y (2018) Reachability judgement in optic ataxia: effect of peripheral vision on hand and target perception in depth. Cortex 98:102-113.

Battaglia-Mayer A, Ferraina S, Genovesio A, Marconi B, Squatrito S, Molinari M, Lacquaniti F, Caminiti R (2001) Eye-hand coordination during reaching. II. An analysis of the relationships between visuomanual signals in parietal cortex and parieto-frontal association projections. Cereb Cortex 11:528-544.

Bosco A, Breveglieri R, Hadjidimitrakis K, Galletti C, Fattori P (2016) Reference frames for reaching when decoupling eye and target position in depth and direction. Sci Rep 6:21646. 
Breveglieri R, Kutz DF, Fattori P, Gamberini M, Galletti C (2002) Somatosensory cells in the parieto-occipital area V6A of the macaque. Neuroreport 13:2113-2116.

Breveglieri R, Galletti C, Gamberini M, Passarelli L, Fattori P (2006) Somatosensory cells in area PEc of macaque posterior parietal cortex. J Neurosci 26:3679-3684.

Breveglieri R, Galletti C, Monaco S, Fattori P (2008) Visual, somatosensory, and bimodal activities in the macaque parietal area PEc. Cereb Cortex 18:806-816.

Breveglieri R, Hadjidimitrakis K, Bosco A, Sabatini SP, Galletti C, Fattori P (2012) Eye position encoding in three-dimensional space: integration of version and vergence signals in the medial posterior parietal cortex. J Neurosci 32:159-169.

Caspari N, Janssens T, Mantini D, Vandenberghe R, Vanduffel W (2015) Covert shifts of spatial attention in the macaque monkey. $J$ Neurosci 35:7695-7714.

Caspari N, Arsenault JT, Vandenberghe R, Vanduffel W (2018) Functional similarity of medial superior parietal areas for shift-selective attention signals in humans and monkeys. Cereb Cortex 28:2085-2099.

Cheney PD, Fetz EE (1980) Functional classes of primate corticomotoneuronal cells and their relation to active force. $J$ Neurophysiol 44:773-791.

Ciavarro M, Ambrosini E, Tosoni A, Committeri G, Fattori P, Galletti C (2013) rTMS of medial parieto-occipital cortex interferes with attentional reorienting during attention and reaching tasks. J Cogn Neurosci 25:1453-1462.

Cui H, Andersen RA (2011) Different representations of potential and selected motor plans by distinct parietal areas. J Neurosci 31:18130-18136.

De Vitis M, Breveglieri R, Hadjidimitrakis K, Vanduffel W, Galletti C, Fattori $P$ (2019) The neglected medial part of macaque area PE: segregated processing of reach depth and direction. Brain Struct Funct 224:2537-2557.

Diomedi S, Vaccari FE, Filippini M, Fattori P, Galletti C (2020) Mixed selectivity in macaque medial parietal cortex during eye-hand reaching. iScience 23:101616.

Duffy FH, Burchfiel JL (1971) Somatosensory system: organizational hierarchy from single units in monkey area 5. Science 172:273275.

Evarts EV (1969) Activity of pyramidal tract neurons during postural fixation. J Neurophysiol 32:375-385.

Fattori P, Breveglieri R, Bosco A, Gamberini M, Galletti C (2017) Vision for prehension in the medial parietal cortex. Cereb Cortex 27:1149-1163.

Ferraina S, Battaglia-Mayer A, Genovesio A, Marconi B, Onorati P, Caminiti R (2001) Early coding of visuomanual coordination during reaching in parietal area PEc. J Neurophysiol 85:462-467.

Ferraina S, Brunamonti E, Giusti MA, Costa S, Genovesio A, Caminiti $R$ (2009) Reaching in depth: hand position dominates over binocular eye position in the rostral superior parietal lobule. J Neurosci 29:11461-11470.

Fluet MC, Baumann MA, Scherberger H (2010) Context-specific grasp movement representation in macaque ventral premotor cortex. J Neurosci 30:15175-15184.

Fromm C (1983) Changes of steady state activity in motor cortex consistent with the length-tension relation of muscle. Pflugers Arch 398:318-323.

Galletti C, Fattori P (2003) Neuronal mechanisms for detection of motion in the field of view. Neuropsychologia 41:1717-1727.

Galletti C, Battaglini PP, Fattori P (1995) Eye position influence on the parieto-occipital area PO (V6) of the macaque monkey. Eur $\mathrm{J}$ Neurosci 7:2486-2501.

Galletti C, Fattori P, Kutz DF, Gamberini M (1999) Brain location and visual topography of cortical area V6A in the macaque monkey. Eur J Neurosci 11:575-582.

Galletti C, Breveglieri R, Lappe M, Bosco A, Ciavarro M, Fattori P (2010) Covert shift of attention modulates the ongoing neural activity in a reaching area of the macaque dorsomedial visual stream. PLoS One 5:e15078.
Gamberini M, Galletti C, Bosco A, Breveglieri R, Fattori P (2011) Is the medial posterior parietal area $\mathrm{V} 6 \mathrm{~A}$ a single functional area? J Neurosci 31:5145-5157.

Gamberini M, Dal Bò G, Breveglieri R, Briganti S, Passarelli L, Fattori $P$, Galletti C (2018) Sensory properties of the caudal aspect of the macaque's superior parietal lobule. Brain Struct Funct 223:18631879.

Gamberini M, Passarelli L, Fattori P, Galletti C (2020) Structural connectivity and functional properties of the macaque superior parietal lobule. Brain Struct Funct 225:1349-1367.

Gardner EP, Babu KS, Reitzen SD, Ghosh S, Brown AS, Chen J, Hall AL, Herzlinger MD, Kohlenstein JB, Ro JY (2007) Neurophysiology of prehension. I. Posterior parietal cortex and object-oriented hand behaviors. J Neurophysiol 97:387-406.

Georgopoulos AP, Caminiti R, Kalaska JF (1984) Static spatial effects in motor cortex and area 5: quantitative relations in a two-dimensional space. Exp brain Res 54:446-454.

Granek JA, Pisella L, Blangero A, Rossetti Y, Sergio LE (2012) The role of the caudal superior parietal lobule in updating hand location in peripheral vision: further evidence from optic ataxia. PLoS One 7:e46619.

Hadjidimitrakis K, Bertozzi F, Breveglieri R, Bosco A, Galletti C, Fattori $P$ (2014a) Common neural substrate for processing depth and direction signals for reaching in the monkey medial posterior parietal cortex. Cereb Cortex 24:1645-1657.

Hadjidimitrakis K, Bertozzi F, Breveglieri R, Fattori P, Galletti C (2014b) Body-centered, mixed, but not hand-centered coding of visual targets in the medial posterior parietal cortex during reaches in 3D space. Cereb Cortex 24:3209-3220.

Hadjidimitrakis K, Dal Bo' G, Breveglieri R, Galletti C, Fattori P (2015) Overlapping representations for reach depth and direction in caudal superior parietal lobule of macaques. J Neurophysiol 114:23402352.

Hamel-Pâquet C, Sergio LE, Kalaska JF (2006) Parietal area 5 activity does not reflect the differential time-course of motor output kinetics during arm-reaching and isometric-force tasks. J Neurophysiol 95:3353-3370.

Impieri D, Zilles K, Niu M, Rapan L, Schubert N, Galletti C, PalomeroGallagher N (2019) Receptor density pattern confirms and enhances the anatomic-functional features of the macaque superior parietal lobule areas. Brain Struct Funct 224:2733-2756.

Kalaska JF (1996) Parietal cortex area 5 and visuomotor behavior. Can J Physiol Pharmacol 74:483-498.

Kalaska JF, Hyde ML (1985) Area 4 and area 5: differences between the load direction-dependent discharge variability of cells during active postural fixation. Exp brain Res 59:197-202.

Kalaska JF, Cohen DA, Prud'homme M, Hyde ML (1990) Parietal area 5 neuronal activity encodes movement kinematics, not movement dynamics. Exp brain Res 80:351-364.

Kelley TA, Serences JT, Giesbrecht B, Yantis S (2008) Cortical mechanisms for shifting and holding visuospatial attention. Cereb Cortex 18:114-125.

Kutz DF, Fattori P, Gamberini M, Breveglieri R, Galletti C (2003) Early- and late-responding cells to saccadic eye movements in the cortical area V6A of macaque monkey. Exp Brain Res 149:83-95.

Kutz DF, Marzocchi N, Fattori P, Cavalcanti S, Galletti C (2005) Realtime supervisor system based on trinary logic to control experiments with behaving animals and humans. J Neurophysiol 93:3674-3686.

Lacquaniti F, Guigon E, Bianchi L, Ferraina S, Caminiti R (1995) Representing spatial information for limb movement: role of area 5 in the monkey. Cereb Cortex 5:391-409.

Luppino G, Ben Hamed S, Gamberini M, Matelli M, Galletti C (2005) Occipital (V6) and parietal (V6A) areas in the anterior wall of the parieto-occipital sulcus of the macaque: a cytoarchitectonic study. Eur J Neurosci 21:3056-3076.

Marzocchi N, Breveglieri R, Galletti C, Fattori P (2008) Reaching activity in parietal area $\mathrm{V} 6 \mathrm{~A}$ of macaque: eye influence on arm activity or retinocentric coding of reaching movements? Eur J Neurosci 27:775-789. 
McGuire LMM, Sabes PN (2011) Heterogeneous representations in the superior parietal lobule are common across reaches to visual and proprioceptive targets. J Neurosci 31:6661-6673.

Mikula L, Blohm G, Koun É, Khan AZ, Pisella L (2021) Movement drift in optic ataxia reveals deficits in hand state estimation in oculocentric coordinates. J Exp Psychol Hum Percept Perform 47:635647.

Molenberghs P, Mesulam MM, Peeters R, Vandenberghe RRC (2007) Remapping attentional priorities: differential contribution of superior parietal lobule and intraparietal sulcus. Cereb Cortex $17: 2703-2712$.

Mountcastle VB, Lynch JC, Georgopoulos A, Sakata H, Acuna C (1975) Posterior parietal association cortex of the monkey: command functions for operations within extrapersonal space. J Neurophysiol 38:871-908.

Pandya DN, Seltzer B (1982) Intrinsic connections and architectonics of posterior parietal cortex in the rhesus monkey. J Comp Neurol 204:196-210.

Piserchia V, Breveglieri R, Hadjidimitrakis K, Bertozzi F, Galletti C, Fattori $P$ (2017) Mixed body/hand reference frame for reaching in
3D space in macaque parietal area PEc. Cereb Cortex 27:19761990.

Raffi M, Ballabeni A, Maioli MG, Squatrito S (2008) Neuronal responses in macaque area PEc to saccades and eye position. Neuroscience 156:413-424.

Rossetti Y, Pisella L, Mclntosh RD (2019) Definition: optic ataxia. Cortex 121:481.

Sakata H, Takaoka Y, Kawarasaki A, Shibutani H (1973) Somatosensory properties of neurons in the superior parietal cortex (area 5) of the rhesus monkey. Brain Res 64:85-102.

Shi Y, Apker G, Buneo CA (2013) Multimodal representation of limb endpoint position in the posterior parietal cortex. J Neurophysiol 109:2097-2107.

Vandenberghe R, Gitelman DR, Parrish TB, Mesulam MM (2001) Functional specificity of superior parietal mediation of spatial shifting. Neuroimage 14:661-673.

Yantis S, Schwarzbach J, Serences JT, Carlson RL, Steinmetz MA, Pekar JJ, Courtney SM (2002) Transient neural activity in human parietal cortex during spatial attention shifts. Nat Neurosci 5:9951002.

Zar JH (1999) Biostatistical analysis. Hoboken: Prentice Hall. 